\title{
Prediction of Alzheimer's disease in subjects with mild cognitive impairment from the ADNI cohort using patterns of cortical thinning
}

\author{
Simon F. Eskildsen, Pierrick Coupé, Daniel García-Lorenzo, \\ Vladimir Fonov, Jens C. Pruessner, D. Louis Collins, and the \\ Alzheimer's Disease Neuroimaging Initiative \\ Neurolmage, Volume 65, 15 January 2013, Pages 511-521 \\ doi: 10.1016/j.neuroimage.2012.09.058 \\ http://www.sciencedirect.com/science/article/pii/S1053811912009755 \\ Copyright 2012 Elsevier Inc. This postprint provided under the terms of a \\ Creative Commons Attribution-NonCommercial-NonDerivative licence.
} protected by copyright with all rights reserved unless otherwise indicated. 
Title: Prediction of Alzheimer's disease in subjects with mild cognitive impairment from the ADNI cohort using patterns of cortical thinning

Authors: Simon F. Eskildsen ${ }^{\mathrm{a}, \mathrm{b},{ }^{*}}$, Pierrick Coupéb,c ${ }^{\mathrm{b}}$, Daniel García-Lorenzo ${ }^{\mathrm{b}, \mathrm{d}}$, Vladimir Fonov ${ }^{\mathrm{b}}$, Jens C. Pruessner $^{\mathrm{e}}, \mathrm{D}$. Louis Collins ${ }^{\mathrm{b}}$ and the Alzheimer's Disease Neuroimaging Initiative**

${ }^{a}$ Center of Functionally Integrative Neuroscience, Aarhus University, Aarhus, Denmark

${ }^{\mathrm{b}}$ McConnell Brain Imaging Centre, Montreal Neurological Institute, McGill University, Montreal, Canada

${ }^{c}$ Laboratoire Bordelais de Recherche en Informatique, Unité Mixte de Recherche CNRS (UMR 5800), Bordeaux, France

${ }^{d}$ Centre de neuro-imagerie de recherhe, Institut du Cerveau et de la Moelle épinière, Paris, France

${ }^{\mathrm{e}}$ Departments of Psychiatry, Neurology and Neurosurgery, McGill University, Montreal, Canada

Word count: 7816 (excluding figure and table captions)

6 tables and 5 figures

*Corresponding author:

Simon Fristed Eskildsen

Center of Functionally Integrative Neuroscience, Aarhus University, Nørrebrogade 44, Aarhus, Denmark

Telephone: +45-7846-9939, mobile: $+45-2210-1234$, fax: $+45-8949-4400$

Email: seskildsen@cfin.au.dk

\footnotetext{
** Data used in the preparation of this article were obtained from the Alzheimer's Disease Neuroimaging Initiative (ADNI) database (www.loni.ucla.edu/ADNI). As such, the investigators within the ADNI contributed to the design and implementation of $A D N I$ and/or provided data but did not participate in analysis or writing of this report. Complete listing of ADNI investigators is available at http://adni.loni.ucla.edu/wpcontent/uploads/how_to_apply/ADNI_Authorship_List.pdf).
} 


\begin{abstract}
Predicting Alzheimer's disease (AD) in individuals with some symptoms of cognitive decline may have great influence on treatment choice and disease progression. Structural magnetic resonance imaging (MRI) has the potential of revealing early signs of neurodegeneration in the human brain and may thus aid in predicting and diagnosing AD. Surface-based cortical thickness measurements from T1-weighted MRI have demonstrated high sensitivity to cortical gray matter changes. In this study we investigated the possibility for using patterns of cortical thickness measurements for predicting AD in subjects with mild cognitive impairment $(\mathrm{MCl})$. We used a novel technique for identifying cortical regions potentially discriminative for separating individuals with $\mathrm{MCl}$ who progress to probable $A D$, from individuals with $\mathrm{MCl}$ who do not progress to probable AD. Specific patterns of atrophy were identified at four time periods before diagnosis of probable $A D$ and features were selected as regions of interest within these patterns. The selected regions were used for cortical thickness measurements and applied in a classifier for testing the ability to predict $A D$ at the four stages. In the validation, the test subjects were excluded from the feature selection to obtain unbiased results. The accuracy of the prediction improved as the time to conversion from $\mathrm{MCl}$ to $\mathrm{AD}$ decreased, from $70 \%$ at 3 years before the clinical criteria for $A D$ was met, to $76 \%$ at 6 months before $A D$. By inclusion of test subjects in the feature selection process, the prediction accuracies were artificially inflated to a range of $73 \%$ to $81 \%$. Two important results emerge from this study. First, prediction accuracies of conversion from $\mathrm{MCl}$ to $\mathrm{AD}$ can be improved by learning the atrophy patterns that are specific to the different stages of disease progression. This has the potential to guide the further development of imaging biomarkers in AD. Second, the results show that one needs to be careful when designing training, testing and validation schemes to ensure that datasets used to build the predictive models are not used in testing and validation.
\end{abstract}

Keywords - AD, MCl, MRI, cortical thickness, prediction. 


\section{Introduction}

The ability to diagnose and predict Alzheimer's disease (AD) at an early or even pre-clinical stage has great impact on the possibility for improving treatment choices of the disease. This may in turn lead to reduced costs associated with long-term care. In addition, accurate prediction may also reduce costs associated with selecting subjects for pharmaceutical trials when performing large scale tests on disease modifying drugs, since false positives can be excluded in the initial stage. $A D$ is characterized by accumulation of amyloid- $\beta(A \beta)$ and hyperphosphorylated tau in the brain, eventually leading to neurodegeneration. To support an early diagnosis of $A D$, various biomarkers are currently being investigated. Even though the accumulation of $A \beta$ can be detected in the cerebrospinal fluid (CSF), or by using positron emission tomography (PET) years before structural changes can be detected, structural imaging markers based on magnetic resonance imaging (MRI) are considered more sensitive to change after the first symptoms appear (Frisoni et al., 2010). Signs of atrophy in the medial temporal lobes may aid in differentiating $A D$ from other pathologies as MRI examinations often are part of the clinical assessment standard of care in patients with mild cognitive impairment $(\mathrm{MCl})$. While studies investigating the usefulness of medial temporal lobe atrophy in the diagnosis of AD are ongoing (Coupe et al., 2012; Cuingnet et al., 2011; Jack et al., 2011; Wolz et al., 2011), the assessment of patterns of cortical thinning across the cerebrum may aid to increase the specificity of the diagnosis for the disease (Apostolova and Thompson, 2008; Vemuri et al., 2008).

The high tissue contrast offered by T1-weighted (T1w) MRI enables accurate structural neuroimaging analysis, which may be used as a possible surrogate biomarker for diagnosing and predicting AD (Ritchie and Lovestone, 2002). However, so far the image processing techniques have not been able to accurately predict future probable $A D$ in patients with $\mathrm{MCl}$ (Cuingnet et al., 2011). Measurements of cortical thickness based on MRI are highly sensitive to small structural changes across the cortex and have been widely used to investigate cortical structural changes and differences in various diseases and disorders, such as dementia, schizophrenia and obsessive compulsive disorder (Lerch et al., 2008; Narr et al., 2005; Shin et al., 2007). However, results from previous studies suggest that cortical thickness measurements do not perform better than other techniques when trying to predict AD in subjects with $\mathrm{MCl}$, yielding accuracies from 56\% to 70\% depending on the technique (Cuingnet et al., 2011; Wolz et al., 2011). Cortical thickness is usually measured at a very high resolution (tens of thousands of points on 
the cerebral cortex). Using such high numbers of measurements in prediction may lead to over-fitting in a discriminatory model. The dimensionality can be reduced by defining regions of interests (ROI) in which measurements are averaged or by transformation of the feature space, such as principal component analysis (Yoon et al., 2007). The latter obstructs any meaningful functional or anatomical interpretation of the features. Defining ROls reduces the inherent noise of high resolution data and reduces the risk of over-fitting. Usually such ROIs are predefined from a structural or functional perspective. However, the pattern of neurodegeneration may not follow standard definitions for anatomical or functional regions, thus such ROls may lead to loss of discriminative information. Therefore, data-driven approaches to select discriminative cortical thickness ROIs, independent of any predefined parcellation, may lead to better prediction results.

Another factor preventing high predictive power is the heterogeneity of images when training a classifier. Usually, a classifier is trained with images from individuals who convert to $A D$ at some future time point and images from individuals who do not convert to $A D$ in the follow-up period. Inherently, there is variability in the degree of neurodegeneration simply due to the variability of when the converters actually convert. This variability affects how well the coefficients of the classifier can be fitted. By homogenizing the images with respect to "time to conversion" and thereby the patterns of atrophy, the classifier may learn the specific pattern and better fit the coefficients of the model.

In practice, a classifier must deal with $\mathrm{MCl}$ patients with variable "time to conversion". Thus, to be clinically applicable, the learned patterns of time-homogenized data must be incorporated into a single classifier identifying the patterns in subjects with unknown status and predict a possible time to conversion. To achieve this, it should first be established if improved classification accuracy can be obtained with time-homogenized data. This is the overall question we address in this paper.

\subsection{Previous work}

Recently, the task of predicting conversion from $\mathrm{MCl}$ to $\mathrm{AD}$ has received a lot of attention, mainly driven by the emergence of large multi-center studies collecting data from hundreds of patients and controls (Ellis et al., 2009; Lovestone et al., 2009; Mueller et al., 2005). Numerous approaches using structural imaging have been suggested (Cho et al., 2012; Chupin et al., 2009; Cuingnet et al., 2011; Davatzikos et al., 2011; Koikkalainen et al., 2011; Misra et al., 2009; Querbes et al., 2009; Westman et al., 2011; Wolz et al., 2011). See Yuan et al. (2009) for a meta-analysis of work prior to 2009. Previously published results indicate that using images obtained on a single scanner yield better prediction accuracies (Plant et al., 2010) than images acquired in multi-center studies (Cuingnet et al., 2011; Wolz et al., 2011). 
However, to be useful from a clinical point of view, an imaging marker should be effective across sites and scanners. Therefore, the evaluation of this marker should be performed on data acquired over a range of sites and scanners. The Alzheimer's Disease Neuroimaging Initiative (ADNI) has made such multi-site data publicly available, and thus has enabled the comparison of predictive accuracy of different methods under similar circumstances. Here, we briefly review work on ADNI data for the prediction of conversion from $\mathrm{MCl}$ to $A D$, i.e. classification of progressive $\mathrm{MCl}$ subjects $(\mathrm{pMCl})$ over $\mathrm{MCl}$ subjects who remain stable (sMCl). Results are summarized in Table 1.

Using baseline data, Chupin et al. (2009) automatically segmented the hippocampus and used the volume in a k-means classifier to predict $\mathrm{MCl}$ conversion to $\mathrm{AD}$ within 18 months. They achieved an overall classification accuracy of $64 \%$. Global hippocampal volume might be prone to large interindividual differences and might not be specific for dementia prediction (Lupien et al., 2007).

Misra et al. (2009) used voxel-based morphometry (VBM) to define highly discriminating regions identified by statistical differences between $\mathrm{pMCl}$ vs. $\mathrm{sMCl}$ using various kernel sizes and number of features. Their highest achieved accuracy of $81.5 \%$ is the best result published to date. However, the low number of $\mathrm{pMCl}(\mathrm{n}=27)$ makes it difficult to compare with other published methods on ADNI data, where at least twice as many $\mathrm{pMCl}$ are included.

Querbes et al. (2009) obtained relatively high accuracy (73\%) with high sensitivity (75\%). They used mean cortical thickness within ROIs, defined by grouped Brodmann areas, combined with age in a linear discriminant analysis (LDA) classifier to select an optimal set of ROIs. These ROIs were used to calculate a thickness index that was used for prediction. Unfortunately, they did not use independent training and test sets: the subjects used for testing were also used in the process of selecting the ROls. By including subjects to be classified in the feature selection, the classification procedure is influenced by the characteristics of the test set and will most likely overestimate the accuracy as discussed later in this paper. Furthermore, the discrepancy in number of $\mathrm{sMCl}$ and $\mathrm{pMCl}$ compared to other studies on ADNI data makes it hard to evaluate the results.

Koikkalainen et al. (2011) also obtained relatively high accuracy (72\%) with high sensitivity (77\%) using tensor-based morphometry (TBM) within automatically selected ROls. As in Querbes et al. (2009), the ROIs were selected using statistical maps of differences between all $\mathrm{pMCl}$ and sMCl subjects, while the classification was validated by constructing training and test sets. Since subjects from the test set were 
used to define the ROls, the training and testing was not completely independent, and their results may be biased and the accuracies are most likely overestimated.

Davatzikos et al. (2011) used voxel-based morphometry (VBM) to predict the conversion. They achieved a classification accuracy of $56 \%$ using the VBM maps, and an accuracy of $62 \%$ by including information of t-tau from CSF samples in a support vector machine (SVM) classifier. Davatzikos and colleagues trained the classifier on patients with $A D$ and cognitively normal $(C N)$ individuals. In $A D$, the atrophy spreads from the medial temporal lobe structures to the rest of the cortex (Braak and Braak, 1991). In addition, it is hypothesized that the atrophy is a non-linear process. Therefore, the characteristic structural traits that discriminate between $A D$ and $C N$ may be less sensitive to separate $\mathrm{pMCl}$ from $\mathrm{sMCl}$. This may explain the relatively low accuracy obtained by Davatzikos and colleagues.

Westman et al. (2011) used predefined cortical thickness regions and subcortical volumes to predict conversion. They combined ADNI data with data from a large European project, called AddNeuroMed (Lovestone et al., 2009), to predict converters in the respective cohorts. For ADNI data, they obtained a low accuracy (58\%), while the accuracy for AddNeuroMed was higher (70\%). It should be noted that the AddNeuroMed data was collected at six different sites, while ADNI data was collected at more than 50 different sites. This may explain the superior prediction accuracy on AddNeuroMed data.

Cuingnet et al. (2011) evaluated the prediction power of ten different structural methods, including hippocampal volume and shape, various VBM approaches, and cortical thickness. Using SVM, they obtained accuracies in the range of $58 \%-71 \%$, but with relatively low sensitivities. The validation was performed by splitting the subjects into equally sized training and test sets, which biases the results to the random selection process. Furthermore, the comparison suffered from many image processing failures leading to different training and test sets for each method evaluated. It should be noted that the image processing pipeline used in Cuingnet et al. (2011) deviates from the pipelines of the originally published methods evaluated in the study. Thus, the results may deviate from what can be obtained using the original methods.

Cho et al. (2012) used noise-filtered cortical thickness, principal component analysis and LDA to classify $\mathrm{pMCl}$ over $\mathrm{sMCl}$. Their classifier performed relatively well (71\% correct rate), but with low sensitivity (63\%). Cho and colleagues used the same samples as in Cuingnet et al. (2011) and obtained similar results. 
Wolz et al. (2011) recently published prediction results using the highest number of $\mathrm{sMCl}$ and $\mathrm{pMCl}$ subjects available to date. Baseline scans of the entire $\mathrm{MCl}$ population from the ADNI cohort were selected and multiple methods were applied to predict the conversion to AD. They also used statistical maps to define ROls for respectively TBM and cortical thickness. Since they included test subjects in their ROI selection step, their results may be biased and the accuracy may be overestimated. In addition, they evaluated hippocampal volume and manifold-based learning as predictors. Accuracies in the range of $56 \%$ - $65 \%$ were obtained using the respective methods, while combining the methods increased the accuracy to $68 \%$. Wolz and colleagues also evaluated their methods using the exact same groups as used in Cuingnet et al. (2011) and obtained superior prediction accuracies. As in Cuingnet et al. (2011), comparison between methods was limited by the fact that $12 \%$ of the subjects failed the cortical thickness estimation. However, this study is so far the most comprehensive work evaluating structural methods for prediction of conversion to $A D$ in subjects with $\mathrm{MCl}$. This and the fact that Wolz et al. obtained very competitive results suggest that their work is state of the art and a good candidate for current and future methods. 
Table 1. Previous work on predicting conversion to AD using structural imaging. Best results (measured as highest accuracy) using only structural imaging are shown for each article. $\mathrm{pMCl}=$ progressive $\mathrm{MCl}, \mathrm{sMCl}=\mathrm{stable} \mathrm{MCl}, \mathrm{Acc}=\mathrm{Accuracy}$, Sen=Sensitivity, Spe=Specificity, AUC=Area Under the receiver operating characteristic Curve.

\begin{tabular}{|c|c|c|c|c|c|c|c|c|}
\hline Article & Structure(s) & Method & $\begin{array}{l}\text { Conversion } \\
\text { period }\end{array}$ & $\begin{array}{l}\mathrm{N}(\mathrm{sMCl}, \\
\mathrm{pMCl})\end{array}$ & Acc & Sen & Spe & $A \cup C$ \\
\hline Cho et al. (2012) & Cortex & Cortical thickness & $0-18$ months & 131,72 & $71 \%$ & $63 \%$ & $76 \%$ & NA \\
\hline $\begin{array}{l}\text { Chupin et al. } \\
\text { (2009) }\end{array}$ & $\begin{array}{l}\text { Hippocampus and } \\
\text { amygdale }\end{array}$ & Atlas based & $0-18$ months & 134,76 & $64 \%$ & $60 \%$ & $65 \%$ & NA \\
\hline $\begin{array}{l}\text { Cuingnet et al. } \\
\text { (2011) }\end{array}$ & Hippocampus & Atlas based & $0-18$ months & 134,76 & $67 \%$ & $62 \%$ & $69 \%$ & NA \\
\hline- & Whole brain & VBM (GM) & - & - & $71 \%$ & $57 \%$ & $78 \%$ & NA \\
\hline- & Cortex & Cortical thickness & - & - & $70 \%$ & $32 \%$ & $91 \%$ & NA \\
\hline $\begin{array}{l}\text { Davatzikos et al. } \\
\text { (2011) }\end{array}$ & Whole brain & VBM & $0-36$ months & 170,69 & $56 \%$ & $95 \%$ & $38 \%$ & $73 \%$ \\
\hline $\begin{array}{l}\text { Koikkalainen et } \\
\text { al. (2011) }\end{array}$ & Whole brain & $\begin{array}{l}\text { TBM, combination } \\
\text { of classifiers }\end{array}$ & $0-36$ months & 215,154 & $72 \%$ & $77 \%$ & $71 \%$ & NA \\
\hline $\begin{array}{l}\text { Misra et al. } \\
\text { (2009) }\end{array}$ & Whole brain & VBM, ROIs & $0-36$ months & 76,27 & $82 \%$ & - & - & $77 \%$ \\
\hline $\begin{array}{l}\text { Querbes et al. } \\
\text { (2009) }\end{array}$ & Cortex & Cortical thickness & $0-24$ months & 50,72 & $73 \%$ & $75 \%$ & $69 \%$ & NA \\
\hline $\begin{array}{l}\text { Westman et al. } \\
\text { (2011) }\end{array}$ & $\begin{array}{l}\text { Cortical and } \\
\text { subcortical }\end{array}$ & $\begin{array}{l}\text { Thickness and } \\
\text { volume }\end{array}$ & $0-12$ months & 256,62 & $58 \%$ & $74 \%$ & $56 \%$ & NA \\
\hline $\begin{array}{l}\text { Wolz et al. } \\
\text { (2011) }\end{array}$ & Hippocampus & Atlas based & $0-48$ months & 238,167 & $65 \%$ & $63 \%$ & $67 \%$ & NA \\
\hline- & Whole brain & TBM & - & - & $64 \%$ & $65 \%$ & $62 \%$ & NA \\
\hline- & Whole brain & Manifold learning & - & - & $65 \%$ & $64 \%$ & $66 \%$ & NA \\
\hline- & Cortex & Cortical thickness & - & - & $56 \%$ & $63 \%$ & $45 \%$ & NA \\
\hline- & Combination & Combination & - & - & $68 \%$ & $67 \%$ & $69 \%$ & NA \\
\hline
\end{tabular}

In general, it seems difficult to obtain high sensitivity in the prediction of conversion to $A D$ among individuals with $\mathrm{MCl}$. Only those methods where the feature selection is dependent on the subsequent test subjects seem to reach sensitivities above $70 \%$ while maintaining high accuracy (Koikkalainen et al., 2011; Querbes et al., 2009). The approach of using the same dataset for selection and selective analysis is also referred to as "double dipping" (Kriegeskorte et al., 2009). To conduct a fair validation, methods 
that rely on statistical maps should avoid double dipping by excluding from the feature generation the test subjects used in the subsequent classifier validation. This includes VBM, TBM and cortical thickness methods, which all operate on the voxel/vertex level and can generate features independent of anatomical or functional parcellations.

Both Davatzikos et al. (2011) and Westman et al. (2011) obtained high sensitivities without a bias, but at the cost of very low specificity. Misra et al. (2009) demonstrated superior classification accuracy without bias, but with very few subjects compared to other methods and no sensitivity was reported. However, the area under the receiver operating characteristic (ROC) curve (AUC) was 77\%, which is relatively high. AUC might be a better metric for evaluating classifiers when sample sizes differ. Unfortunately, most studies do not report AUC.

An explanation for the limited predictive power in current methods may be the heterogeneity of the data. Most studies label $\mathrm{MCl}$ patients as progressive or stable according to whether they converted to $A D$ within the time frame they were followed. This time frame varies from study to study and seems, at least for studies on ADNI data, to depend on when the analysis was carried out. By including $\mathrm{MCl}$ patients, who convert to $A D$ in a time period ranging from a few months to three years, disease stage specific patterns maybe missed and lead to reduced sensitivity.

In the present study we are striving to homogenize the data as much as possible by subdividing the $\mathrm{pMCl}$ patients with regard to "time to conversion". This way we are investigating if the predictive power can be improved by focusing on disease stage specific atrophy patterns. In addition, to avoid double dipping, we exclude test subjects from the group used to generate features for the classification. Finally, we apply a stable and robust image processing pipeline to avoid the high exclusion rates reported in previous studies on cortical thickness (Cuingnet et al., 2011; Wolz et al., 2011).

We aim to $i$ ) evaluate if prediction accuracies can be improved by subdividing $\mathrm{MCl}$ subjects, ii) investigate the effect double dipping have on accuracy, and iii) test the robustness of our image processing pipeline for cortical surface extraction. 


\section{Methods}

\subsection{Subjects and acquisition}

All data used in the preparation of this article were obtained from the ADNI database (www.loni.ucla.edu/ADNI). The ADNI was launched in 2003 by the National Institute on Aging, the National Institute of Biomedical Imaging and Bioengineering, the Food and Drug Administration, private pharmaceutical companies and non-profit organizations, as a \$60 million, 5-year public-private partnership. The primary goal of $\mathrm{ADNI}$ has been to test whether serial MRI, positron emission tomography, other biological markers, and clinical and neuropsychological assessment can be combined to measure the progression of $\mathrm{MCl}$ and early $\mathrm{AD}$. Determination of sensitive and specific markers of very early $A D$ progression is intended to aid researchers and clinicians to develop new treatments and monitor their effectiveness, as well as lessen the time and cost of clinical trials.

The ADNI database contains $1.5 \mathrm{~T}$ and 3.0T T1w MRI scans for $\mathrm{AD}, \mathrm{MCl}$, and cognitively normal controls (CN) at several time points. The aim was to follow and collect scans from $\mathrm{MCl}$ patients at baseline, 6 months, 12 months, 18 months, 24 months, 36 months and 48 months. The number of participating subjects was reduced over the course of the study due to drop outs. At each time point a clinical diagnosis was made to identify $\mathrm{MCl}$ subjects who converted to probable AD according to the NINCDSADRDA Alzheimer's Criteria (McKhann et al., 1984).

As reviewed previously, several studies have used the ADNI data to predict which $\mathrm{MCl}$ patients would convert to probable AD using a single MRI scan (Cho et al., 2012; Chupin et al., 2009; Cuingnet et al., 2011; Davatzikos et al., 2011; Koikkalainen et al., 2011; Misra et al., 2009; Querbes et al., 2009; Westman et al., 2011; Wolz et al., 2011). All of these studies have used baseline data for the analysis, which rendered the group of $\mathrm{pMCl}$ heterogeneous with respect to "time to conversion", since the $\mathrm{pMCl}$ patients would convert anytime over the course of 6 months to 4 years follow-up. Such heterogeneity may conceal the specific neurodegenerative processes that may be attributed to the different substages of the disease. For example, the pattern of atrophy may be different in patients one year before diagnosis compared to the pattern two years earlier. In this study, we therefore utilized the full ADNI database and selected scans at various intervals prior to diagnosis. We selected $\mathrm{pMCl}$ scans six months, 12 months, 24 months and 36 months prior to $A D$ diagnosis and grouped these into time-homogeneous groups of $\mathrm{pMCl}$. To identify characteristic traits for disease progression in the $\mathrm{pMCl}$ groups and determine if these could be used as markers for prediction, we compared with the group of stable $\mathrm{MCl}$ 
(sMCl) patients who did not have a change of diagnosis over the course of the ADNI study. Our sMCl group only included those $\mathrm{MCl}$ patients who were followed for at least three years. Thus, for the $\mathrm{sMCl}$ group, we selected scans at baseline.

To compare the classification of the $\mathrm{MCl}$ substages to the more conventional approach of pooling converters, we categorized the $\mathrm{MCl}$ subjects into converters and stables as done in Wolz et al. (2011), where converters were defined as those that had converted as of July 2011 and the complementary MCl subjects constituted the $\mathrm{sMCl}$ group. In this case only baseline scans were used in the classification. This enabled us to evaluate our method in a head to head comparison with the methods published in Wolz et al. (2011).

Table 2 lists the selected $\mathrm{MCl}$ groups after removing subjects due to scanner acquisition artefacts or image processing problems. Fifty-one out of $1872(2.7 \%)$ scans were removed this way (see section "Image processing" below). No differences in age were found between the groups, while all pMCl groups had significantly (t-test, $\mathrm{p}<0.05)$ smaller MMSE scores than the corresponding sMCl group. Lists of subject IDs for all $\mathrm{MCl}$ groups are disclosed in the supplemental material Tables S1 - S7.

Table 2. Demographics of the study groups. Significant differences (t-test, $\mathrm{p}<0.05$ ) for the respective progressive $\mathrm{MCl}(\mathrm{pMCl})$ groups compared to the corresponding stable $\mathrm{MCl}(\mathrm{sMCl})$ group are in boldface.

\begin{tabular}{|l|l|l|l|}
\hline Group & N (females) & Age \pm sd & MMSE \pm sd \\
\hline sMCl & $134(40)$ & $75.0 \pm 7.5$ & $27.6 \pm 1.8$ \\
\hline pMCl6 & $122(47)$ & $75.3 \pm 7.6$ & $\mathbf{2 5 . 3} \pm 2.6$ \\
\hline pMCl12 & $128(54)$ & $75.6 \pm 7.1$ & $\mathbf{2 6 . 0 \pm 2 . 3}$ \\
\hline pMCl24 & $61(24)$ & $74.4 \pm 7.2$ & $\mathbf{2 6 . 7 \pm 1 . 8}$ \\
\hline pMCl36 & $29(13)$ & $75.8 \pm 6.4$ & $\mathbf{2 6 . 9} \pm 1.7$ \\
\hline sMCl + & $227(76)$ & $74.9 \pm 7.7$ & $27.2 \pm 2.5$ \\
\hline pMCl† & $161(64)$ & $74.5 \pm 7.2$ & $\mathbf{2 6 . 4 \pm 2 . 0}$ \\
\hline
\end{tabular}

+ Cohort defined as described in Wolz et al. (2011)

In addition, baseline data for AD patients $(n=194)$ and age-matched cognitive normal (CN) subjects $(n=226)$ were selected to compare prediction rates to classifications of probable AD over CN. All scans used in the study were T1w image volumes acquired on 1.5T MRI scanners. 


\subsection{Image processing}

Images were denoised (Coupe et al., 2008) using an estimated standard deviation of noise (Coupe et al., 2010), bias field corrected (Sled et al., 1998), registered to MNI space (Collins et al., 1994) and skull stripped (Eskildsen et al., 2011a). Cortical thickness was calculated using FACE (fast accurate cortex extraction) (Eskildsen and Ostergaard, 2006) and mapped to the cortical surface of a population-specific average non-linear anatomical template (Fonov et al., 2011). Cortical segmentations were manually checked for errors by an expert and subjects were excluded if errors were found in one of the image processing steps mentioned above. The quality control removed only $2.7 \%$ of the scans processed, resulting in the group sizes listed in Table 2. In general, the scans were excluded due to image artefacts $(n=5)$, insufficient stereotaxic registration $(n=7)$ and insufficient skull stripping ( $n=39)$.

\subsection{Feature generation}

In the experiments below, we use a leave-one-out (LOO) validation strategy where for each comparison (e.g., $\mathrm{pMCl} 12$ vs $\mathrm{sMCl}$ ), all the subjects (the ensemble of all $\mathrm{pMCl} 12$ and $\mathrm{sMCl}$ ) except one are used to select features and generate a classification model and the one subject left out is then used for testing. This procedure is repeated for every subject in the two groups compared, thus validating the method with every subject. Since the test subject is not used in the selection of features, nor in building the classifier, we avoid any bias or "double dipping" in our efforts of predicting converters. It is important to note that we obtain a unique set of features and classifier for each LOO test, for a total of 876 feature sets for the $\mathrm{MCl}$ stages classification and 388 for the $\mathrm{pMCl}-\mathrm{sMCl}$ classification with the groups defined as in Wolz et al. (2011). See Fig. 1 for an overview of the feature generation, selection and subject classification.

To explore the patterns of atrophy at the different stages of the progression from $M C l$ to $A D$, we devised a data-driven feature selection method for classification. Using the training sets (i.e., the groups to be compared, less the subject to be tested), statistical parametric maps of differences in cortical thickness between the $\mathrm{sMCl}$ group and the $\mathrm{pMCl}$ groups were constructed by one-sided t-tests per vertex of the template surface (from a total of 162,582 vertices). Our aim was to generate a compact set of features with high discriminating power. Candidate features were restricted to a proportion, $\phi$, of the cortical surface with the largest magnitude t-values. Within this thresholded t-map (Fig. 2a), local maxima were detected and used as seed points for a constrained region growing algorithm. For each maxima, region growing were performed downhill only in a circular fashion constraining the area to a maximum of 300 $\mathrm{mm}^{2}$ and a maximum accumulated $\mathrm{t}$-value of $\delta$, where $\delta$ is defined as 


$$
\delta=\frac{\sum_{t \in \phi} t}{c}
$$

where $c$ is the number of seed points. Only regions that reached an accumulated t-value of $\delta$ were kept. These regions constituted the candidate ROIs (Fig. 2b). The proportion, $\phi$, of the cortical surface is used to guide the region growing algorithm and limiting the number of seed points. However, restricting the features to a too small proportion of the surface leads to very small patches of cortical thickness which are more affected by noise. In our experiments we found $\phi$ in the range of $10 \%-15 \%$ to be a suitable trade-off (see Fig. 3). In the remaining experiments we therefore set $\phi=15 \%$.

Cortical features were determined as the mean cortical thickness for each ROI. To remove redundant features and keep discriminant features, we used the minimal-redundancy-maximal-relevance (mRMR) criterion (Peng et al., 2005) as feature selection. We used the mutual information difference metric and discretized the features to three states $(-1,0,1)$ by thresholding at $\mu \pm \sigma$. This was done to ease the mutual information computation as described in (Peng et al., 2005). From our experiments (Fig. 3), we empirically chose to keep the 10 best features according to the mRMR criterion. It should be noted that this number may not be optimal for all classification problems. 


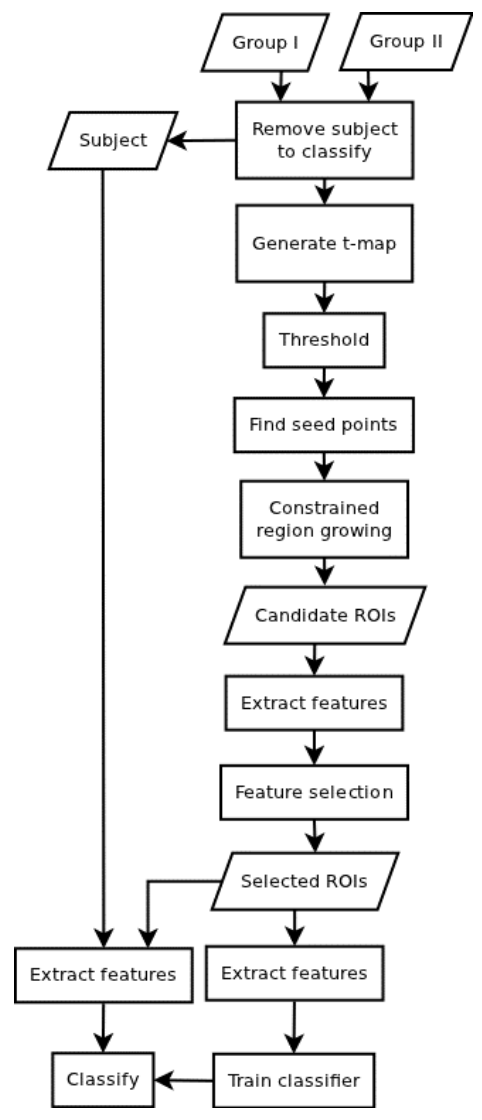

Figure 1. Flow chart illustrating the process of generating and selecting features without being biased by the subject to classify. This procedure is repeated for each subject in each group when performing the leave-one-out validation.

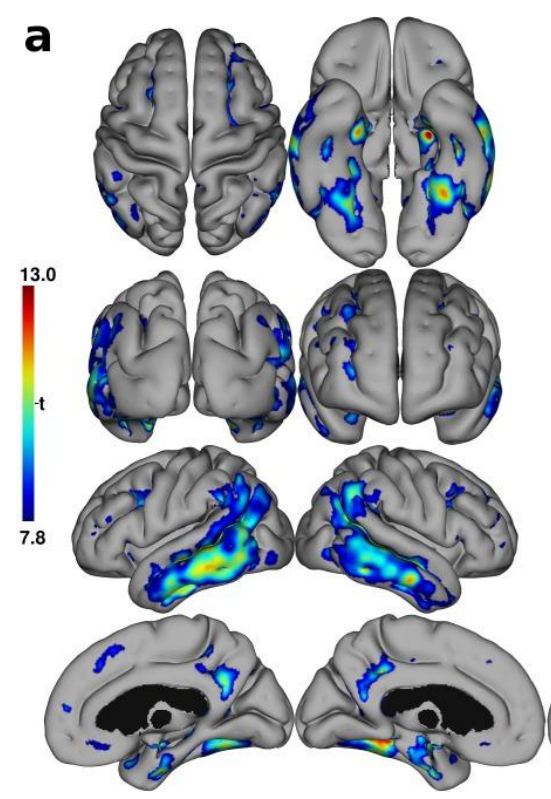

b
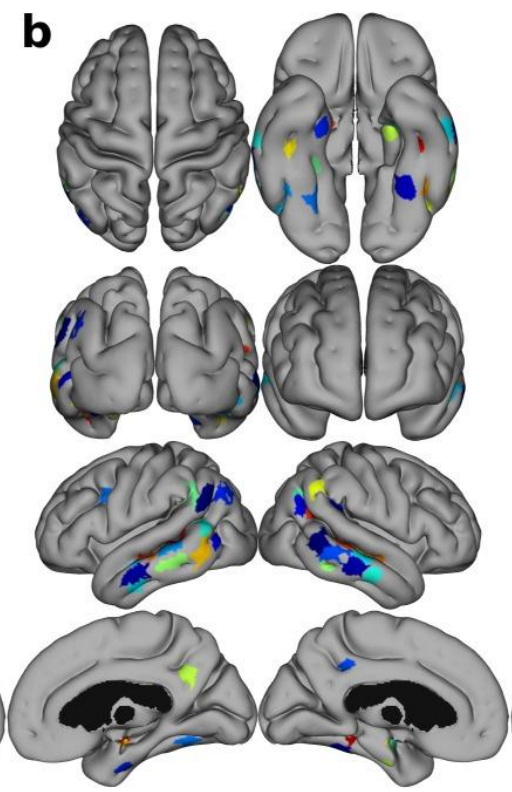

C
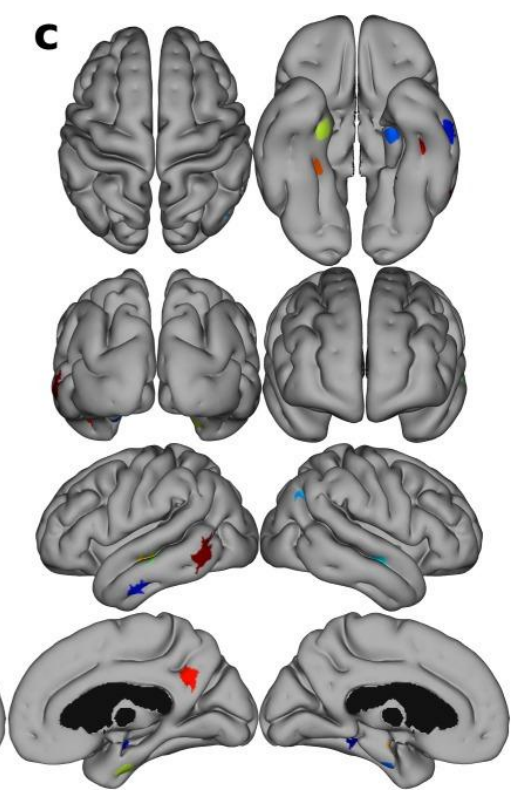

Figure 2. A single instance of the feature generation and selection process for the $A D$ vs CN classification showing a) thresholded t-map, b) ROIs after constrained region growing from seed points, and c) ROls left after feature selection. 


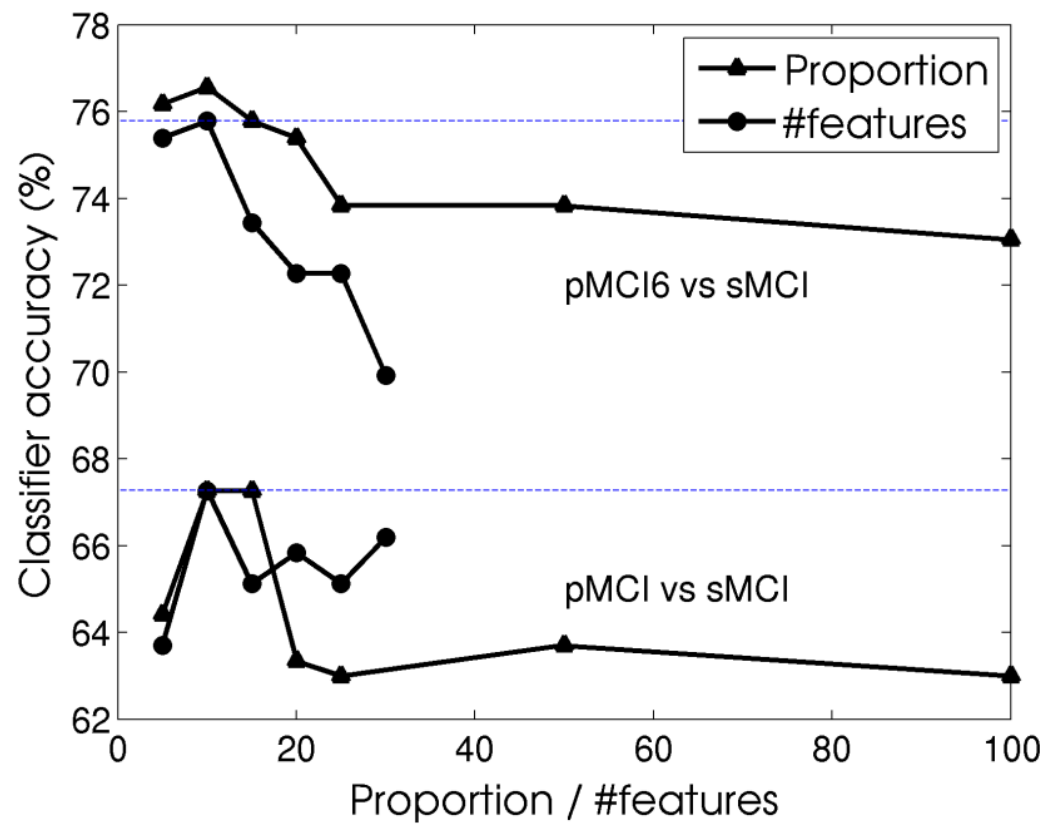

Figure 3. Experimental results of varying the proportion of the cortical surface used for finding candidate features and varying the number of features selected by the mRMR criterion. For variations in proportion, the number of features was fixed at 10 . For variations in number of features, the proportion was fixed at $\mathbf{1 5 \%}$. The dashed lines mark the classifier accuracies reported in Table 4 for the respective prediction problems.

\subsection{Classification}

LDA was used for the classification. As illustrated in Fig. 1, for each subject to classify a separate classifier was trained with the remaining subjects using the subject-specific feature set. The correct classification rate, the sensitivity, the specificity, and the AUC were calculated from the results. Differences in AUC between classifiers were tested using a non-parametric method (DeLong et al., 1988) implemented by the open-source R library, pROC (Robin et al., 2011). Classifier differences for the same classification problem were evaluated by two-sided, paired tests, while differences across classification problems were evaluated by two-sided, unpaired tests. Furthermore, McNemar's chi-square test was used to assess whether the classification performed better than a random classifier. This was done by performing a random classification and calculating the p-value of McNemar's test with significance level at 0.05 that our classification was better than the random classification. This validation process was repeated 1000 times for each of the 1264 LOO experiments and the median p-value was reported.

We compared the results with results obtained using double dipping, i.e., where the feature generation and selection included the subject under study as done in several previous studies (Koikkalainen et al., 2011; Querbes et al., 2009; Wolz et al., 2011). This means that only one set of features was generated for each classification problem. The classifications were still done as LOO experiments. 
Since we wanted to evaluate the prediction power of a single MRI examination, other important features, such as CSF Tau and A $\beta$ markers and ApoE genotyping, were not included in the classifiers, as they are often not available in population samples. However, we did include age as feature in the classifiers, as age is an important associated risk factor in $A D$ and is costless to generate.

Anatomical regions included in the automatically selected ROIs where found using the labels described by Tzourio-Mazoyer et al. (2002) adapted to a surface based atlas matching our template surface. The likelihood $\mathscr{L}$ for an anatomical label being involved in a classification problem was calculated as:

$$
\mathscr{L}(l)=\sum_{v \in l} p(v)
$$

where $l$ is the anatomical label, $v$ is a vertex of the template surface belonging to $l$, and

$$
p(v)=\frac{1}{n} \sum_{i}^{n} x_{i}(v), \text { where } x(v)=\left\{\begin{array}{l}
1, \text { if selected } \\
0, \text { otherwise }
\end{array}\right.
$$

where $n$ is the number of LOO experiments and $x$ is a binary function determining if $v$ is selected in a particular experiment. $\mathscr{L}$ is an expression of how often an anatomical region is included in the classification problem and counting the area of involvement. Note that the automatically selected ROIs have arbitrary borders, thus a single ROI may span several anatomical regions with varying amount of involvement. To avoid favouring large anatomical labels, we standardized the likelihood scores by estimations of the likelihood distribution of each label if features were randomly selected. Thus, we performed permutation tests of the LOO experiments by randomizing the position of the features and calculated $\mathscr{L}$. This was repeated 500 times to get estimators for the distributions of $\mathscr{L}$ for each label in the different experiments. Finally, from these estimators we calculated standardized likelihood scores (likelihood z scores).

\subsection{Prediction}

As a proof of principle we investigated the prediction accuracy in a clinically relevant experiment, where baseline scans of $\mathrm{MCl}$ subjects were evaluated by each of the four stratified classifiers, carefully removing any duplicates from the training sets. Based on the combined maximum posterior probability from the four classifiers, each baseline scan was classified into the categories: $\mathrm{pMCl6,pMCl} 12, \mathrm{pMCl} 24$, $\mathrm{pMCl} 36$, and sMCl. Only scans of subjects where the status was known after three years from baseline were included in this test $(\mathrm{N}=283)$. The result was compared to the true conversion time. 


\section{Results}

Table 3 lists the regions of cortical thickness chosen most often by the feature selection for each classifier sorted by likelihood z score. These regions are also shown in the supplemental material, Figs. S1 - S6. Medial temporal lobe structures were chosen in all classification problems. However, the hippocampus, which is considered to be affected in the incipient stages of AD (Braak and Braak, 1991), was not chosen in the $\mathrm{pMCl} 36$ classification problem.

Classification performances for the six classification problems using both independent and dependent (i.e., "double dipping") feature sets are shown in Table 4. Using the dependent feature sets, we obtain higher accuracies and AUCs in all classification problems. The ROC curves for the classifications are shown in Fig. 4. The figure shows how the double dipping approach has a huge bias on the performance of the classifier, which can be seen by the larger distance from the curves to the diagonal marking the random guess. Furthermore, the order of "time to conversion" is clearly more consistent with the independent features; $\mathrm{pMCl} 36$ prediction is worst, $\mathrm{pMCl} 24$ second worst, and so on.

Table 5 lists classification results after including age in the LDA. Not all accuracies are improved by adding age information. However, all AUCs are improved except for the conventional classification of $\mathrm{pMCl}$ vs. $\mathrm{sMCl}$, which is slightly reduced after adding age information. None of the $\mathrm{pMCl}$ classifiers are significantly $(p>0.06)$ improved by adding age information. P-values for testing the true differences in AUC across classification problems are given in Table 6. The AD vs. CN classifier is significantly $(p<0.001)$ better than the other classifiers as expected. The short-term prediction classifiers (<12 months) are significantly better than the conventional baseline grouping of $\mathrm{pMCl}$ and $\mathrm{sMCl}$, while the differences in AUC between the long-term prediction classifiers ( $>12$ months) and the conventional grouping did not reach significance. 
Table 3. Anatomical regions most often selected by the independent feature selection algorithm for each classification problem. Showing regions with a likelihood $\mathrm{z}$ score $>\mathbf{1 0}$ for each hemisphere. Medial temporal lobe structures are in boldface.

\begin{tabular}{|c|c|c|c|c|}
\hline Classification & Left hemisphere & Likelihood z & Right hemisphere & Likelihood z \\
\hline pMCl36 vs sMCl & $\begin{array}{l}\text { Lingual gyrus } \\
\text { Inferior orbitofrontal } \\
\text { Supramarginal gyrus } \\
\text { Middle cingulate gyrus } \\
\text { Superior temporal gyrus } \\
\text { Gyrus rectus } \\
\text { Calcarine sulcus }\end{array}$ & $\begin{array}{l}36 \\
32 \\
31 \\
23 \\
18 \\
14 \\
13\end{array}$ & $\begin{array}{l}\text { Para-hippocampal gyrus } \\
\text { Superior temporal gyrus } \\
\text { Lingual gyrus }\end{array}$ & $\begin{array}{l}34 \\
20 \\
16\end{array}$ \\
\hline $\mathrm{pMCl} 24$ vs sMCl & $\begin{array}{l}\text { Olfactory cortex } \\
\text { Middle occipital gyrus } \\
\text { Superior occipital gyrus } \\
\text { Hippocampus } \\
\text { Cuneus } \\
\text { Insula } \\
\text { Inferior frontal opercularis }\end{array}$ & $\begin{array}{l}52 \\
30 \\
28 \\
21 \\
18 \\
13 \\
11\end{array}$ & $\begin{array}{l}\text { Para-hippocampal gyrus } \\
\text { Middle temporal pole } \\
\text { Hippocampus } \\
\text { Supramarginal gyrus } \\
\text { Precuneus }\end{array}$ & $\begin{array}{l}33 \\
20 \\
19 \\
15 \\
13\end{array}$ \\
\hline pMCl12 vs sMCl & $\begin{array}{l}\text { Superior temporal pole } \\
\text { Middle occipital gyrus } \\
\text { Para-hippocampal gyrus } \\
\text { Hippocampus } \\
\text { Precuneus } \\
\text { Cuneus } \\
\text { Inferior occipital gyrus } \\
\text { Supramarginal gyrus }\end{array}$ & $\begin{array}{l}87 \\
86 \\
81 \\
35 \\
29 \\
27 \\
19 \\
18\end{array}$ & $\begin{array}{l}\text { Amygdala } \\
\text { Hippocampus } \\
\text { Middle temporal pole } \\
\text { Para-hippocampal gyrus } \\
\text { Middle occipital gyrus } \\
\text { Heschl's gyrus } \\
\text { Precuneus } \\
\text { Middle temporal gyrus } \\
\text { Cuneus } \\
\text { Rolandic operculum }\end{array}$ & $\begin{array}{r}149 \\
100 \\
89 \\
71 \\
60 \\
51 \\
44 \\
38 \\
38 \\
27\end{array}$ \\
\hline pMCI6 vs sMCl & $\begin{array}{l}\text { Amygdala } \\
\text { Inferior frontal opercularis } \\
\text { Middle occipital gyrus } \\
\text { Inferior temporal gyrus }\end{array}$ & $\begin{array}{l}44 \\
32 \\
28 \\
23\end{array}$ & $\begin{array}{l}\text { Hippocampus } \\
\text { Para-hippocampal gyrus } \\
\text { Supramarginal gyrus } \\
\text { Precuneus }\end{array}$ & $\begin{array}{l}61 \\
41 \\
34 \\
24\end{array}$ \\
\hline$A D$ vs $C N$ & $\begin{array}{l}\text { Middle temporal gyrus } \\
\text { Inferior temporal gyrus } \\
\text { Hippocampus }\end{array}$ & $\begin{array}{l}124 \\
114 \\
112\end{array}$ & $\begin{array}{l}\text { Fusiform gyrus } \\
\text { Hippocampus } \\
\text { Para-hippocampal gyrus }\end{array}$ & $\begin{array}{l}75 \\
73 \\
61\end{array}$ \\
\hline
\end{tabular}




\begin{tabular}{|l|l|r|l|r|}
\hline & Para-hippocampal gyrus & 99 & Middle temporal gyrus & 36 \\
& Fusiform gyrus & 77 & Precuneus & 32 \\
& & Superior temporal gyrus & 29 \\
\hline $\mathrm{pMCl}^{\text {a }} \mathrm{vs} \mathrm{sMCl}^{\mathrm{a}}$ & Superior temporal pole & 60 & Calcarine sulcus & 73 \\
& Inferior frontal opercularis & 46 & Hippocampus & 50 \\
& Supplemental motor area & 37 & Para-hippocampal gyrus & 36 \\
& Amygdala & 25 & Precuneus & 25 \\
& Para-hippocampal gyrus & 25 & Middle temporal gyrus & 20 \\
& Inferior temporal gyrus & 21 & Fusiform gyrus & 17 \\
& Medial superior frontal gyrus & 21 & & 16 \\
& Precuneus & 12 & & \\
& Middle occipital gyrus & &
\end{tabular}

${ }^{a}$ Cohort defined as in (Wolz et al., 2011)

Table 4. Classification results using independent and dependent feature sets based on cortical thickness features only. $A c c=A c c u r a c y$, Sen=Sensitivity, Spe=Specificity, AUC=Area under the ROC curve, $\mathrm{Cl}=95 \%$ Confidence Interval.

\begin{tabular}{|c|c|c|c|c|c|c|c|c|c|c|}
\hline & \multicolumn{5}{|c|}{ Independent feature sets } & \multicolumn{5}{|c|}{ Dependent feature sets } \\
\hline Classification & $\begin{array}{l}\text { Acc } \\
(\%)\end{array}$ & $\begin{array}{l}\text { Sen } \\
(\%)\end{array}$ & $\begin{array}{l}\text { Spe } \\
\text { (\%) }\end{array}$ & $\begin{array}{c}A U C[\mathrm{Cl}] \\
(\%)\end{array}$ & $\begin{array}{l}\text { McNemar's } \\
\text { test }\end{array}$ & $\begin{array}{l}\text { Acc } \\
(\%)\end{array}$ & $\begin{array}{l}\text { Sen } \\
(\%)\end{array}$ & $\begin{array}{l}\text { Spe } \\
(\%)\end{array}$ & $\begin{array}{c}\text { AUC [CI] } \\
(\%)\end{array}$ & $\begin{array}{l}\text { McNemar's } \\
\text { test }\end{array}$ \\
\hline $\begin{array}{l}\text { pMCl36 vs } \\
\text { sMCl }\end{array}$ & 69.9 & 55.2 & 73.1 & $\begin{array}{c}63.5 \\
{[51.5-} \\
75.5]\end{array}$ & $p=0.198$ & 77.3 & 69.0 & 79.1 & $\begin{array}{c}83.5 \\
{[76.3-} \\
90.6]\end{array}$ & $p<0.001$ \\
\hline $\begin{array}{l}\mathrm{pMCl} 24 \text { vs } \\
\mathrm{sMCl}\end{array}$ & 66.7 & 59.0 & 70.2 & $\begin{array}{c}67.3 \\
{[59.2-} \\
75.4]\end{array}$ & $p=0.062$ & 73.0 & 69.4 & 74.6 & $\begin{array}{c}80.3 \\
{[73.6-} \\
86.9]\end{array}$ & $p<0.001$ \\
\hline $\begin{array}{l}\text { pMCl12 vs } \\
\text { sMCl }\end{array}$ & 72.9 & 75.8 & 70.2 & $\begin{array}{c}76.2 \\
{[70.3-} \\
82.0]\end{array}$ & $p<0.001$ & 74.5 & 75.2 & 73.9 & $\begin{array}{c}81.3 \\
{[76.1-} \\
86.5]\end{array}$ & $p<0.001$ \\
\hline $\begin{array}{l}\mathrm{pMCl} 6 \text { vs } \\
\mathrm{sMCl}\end{array}$ & 75.8 & 75.4 & 76.1 & $\begin{array}{c}80.9 \\
{[75.5-} \\
86.3]\end{array}$ & $p<0.001$ & 80.9 & 78.7 & 82.8 & $\begin{array}{c}84.9 \\
{[80.1-} \\
89.7]\end{array}$ & $p<0.001$ \\
\hline$A D$ vs $C N$ & 84.5 & 79.4 & 88.9 & $\begin{array}{c}90.5 \\
{[87.6-} \\
93.5]\end{array}$ & $p<0.001$ & 86.7 & 80.4 & 92.0 & $\begin{array}{c}91.7 \\
{[89.0-} \\
94.5]\end{array}$ & $p<0.001$ \\
\hline $\begin{array}{l}\text { pMCl' vs } \\
\text { sMCl' }\end{array}$ & 67.3 & 65.8 & 68.3 & $\begin{array}{c}68.5 \\
{[63.0-} \\
74.0]\end{array}$ & $p<0.001$ & 71.1 & 72.1 & 70.5 & $\begin{array}{c}72.7 \\
{[67.5-} \\
77.8]\end{array}$ & $p<0.001$ \\
\hline
\end{tabular}

+ Cohort defined as in (Wolz et al., 2011) 
Table 5. Classification results using independent and dependent feature sets. Classifications based on cortical thickness and age. Acc=Accuracy, Sen=Sensitivity, Spe=Specificity, AUC=Area under the ROC curve, $\mathrm{Cl}=95 \%$ Confidence Interval.

\begin{tabular}{|c|c|c|c|c|c|c|c|c|c|c|}
\hline \multirow[b]{2}{*}{ Classification } & \multicolumn{5}{|c|}{ Independent feature sets } & \multicolumn{5}{|c|}{ Dependent feature sets } \\
\hline & $\begin{array}{l}\text { Acc } \\
(\%)\end{array}$ & $\begin{array}{l}\text { Sen } \\
(\%)\end{array}$ & $\begin{array}{l}\text { Spe } \\
(\%)\end{array}$ & $\begin{array}{c}\text { AUC [CI] } \\
(\%)\end{array}$ & $\begin{array}{c}\text { McNemar's } \\
\text { test }\end{array}$ & $\begin{array}{l}\text { Acc } \\
(\%)\end{array}$ & $\begin{array}{l}\text { Sen } \\
(\%)\end{array}$ & $\begin{array}{l}\text { Spe } \\
(\%)\end{array}$ & $\begin{array}{c}\text { AUC [Cl] } \\
(\%)\end{array}$ & $\begin{array}{c}\text { McNemar's } \\
\text { test }\end{array}$ \\
\hline $\begin{array}{l}\mathrm{pMCl} 36 \text { vs } \\
\mathrm{sMCl}\end{array}$ & 72.4 & 48.3 & 77.6 & $\begin{array}{l}63.7 \\
{[51.5-} \\
75.9]\end{array}$ & $p<0.001$ & 79.1 & 72.4 & 80.6 & $\begin{array}{l}85.4 \\
{[78.4-} \\
92.4]\end{array}$ & $\mathrm{p}<0.001$ \\
\hline $\begin{array}{l}\mathrm{pMCl} 24 \mathrm{vs} \\
\mathrm{sMCl}\end{array}$ & 67.2 & 55.7 & 72.4 & $\begin{array}{l}70.7 \\
{[62.7-} \\
78.7]\end{array}$ & $p=0.001$ & 75.4 & 70.5 & 77.6 & $\begin{array}{l}82.0 \\
{[75.7-} \\
88.3]\end{array}$ & $p<0.001$ \\
\hline $\begin{array}{l}\mathrm{pMCl} 12 \text { vs } \\
\mathrm{sMCl}\end{array}$ & 70.6 & 72.7 & 68.7 & $\begin{array}{l}76.3 \\
{[70.4-} \\
82.1]\end{array}$ & $p<0.001$ & 74.1 & 76.6 & 71.6 & $\begin{array}{l}82.0 \\
{[76.9-} \\
87.0]\end{array}$ & $p<0.001$ \\
\hline $\begin{array}{l}\text { pMCl6 vs } \\
\text { sMCl }\end{array}$ & 74.6 & 72.1 & 76.9 & $\begin{array}{l}81.1 \\
{[75.8-} \\
86.5]\end{array}$ & $p<0.001$ & 78.9 & 77.1 & 80.6 & $\begin{array}{l}86.0 \\
{[81.4-} \\
90.7]\end{array}$ & $p<0.001$ \\
\hline$A D$ vs $C N$ & 85.5 & 80.4 & 89.8 & $\begin{array}{l}92.0 \\
{[89.3-} \\
94.7]\end{array}$ & $p<0.001$ & 87.4 & 82.5 & 91.6 & $\begin{array}{l}93.1 \\
{[90.7-} \\
95.6]\end{array}$ & $p<0.001$ \\
\hline $\begin{array}{l}\text { pMClt vs } \\
\text { sMCl' }\end{array}$ & 67.8 & 64.6 & 70.0 & $\begin{array}{l}68.2 \\
{[62.7-} \\
73.6]\end{array}$ & $p<0.001$ & 68.3 & 67.7 & 68.7 & $\begin{array}{l}74.7 \\
{[69.8-} \\
79.7]\end{array}$ & $p<0.001$ \\
\hline
\end{tabular}

+ Cohort defined as in (Wolz et al., 2011) 

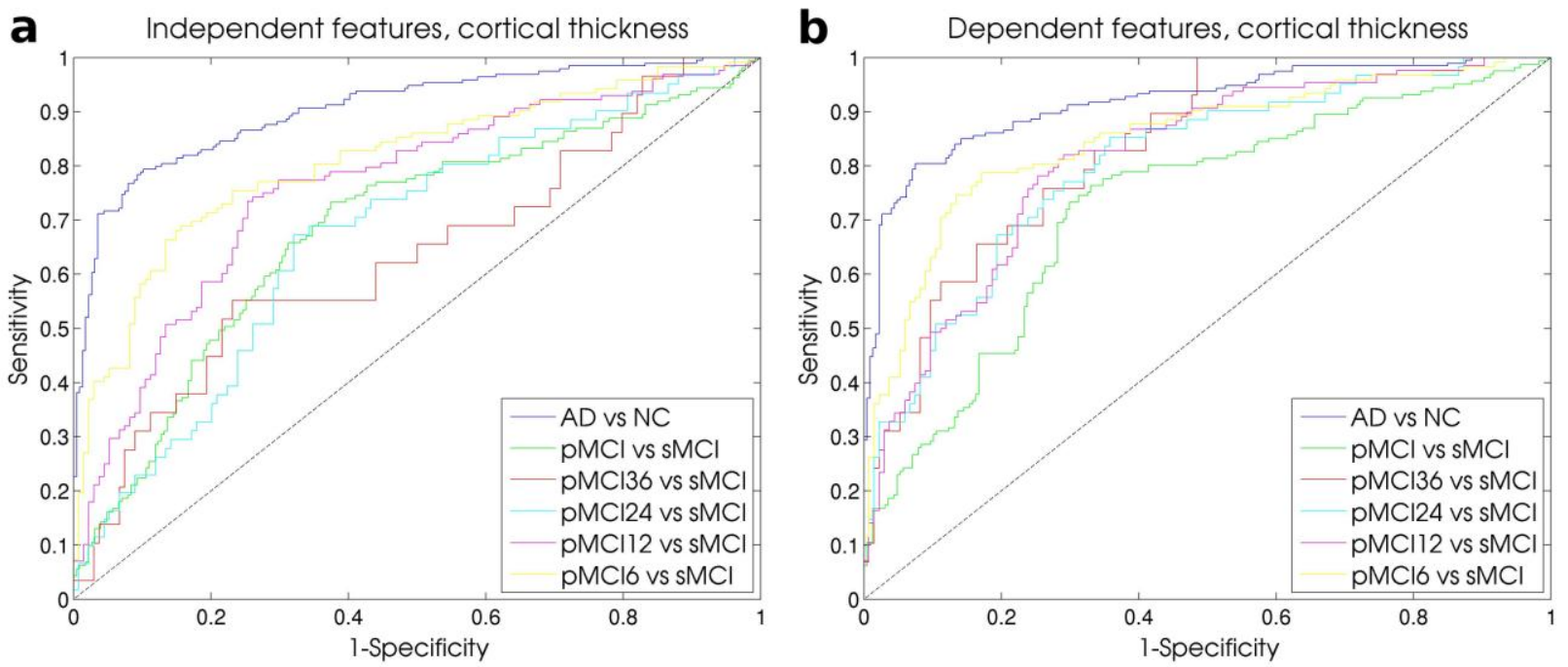

C Independent features, cortical thickness and age
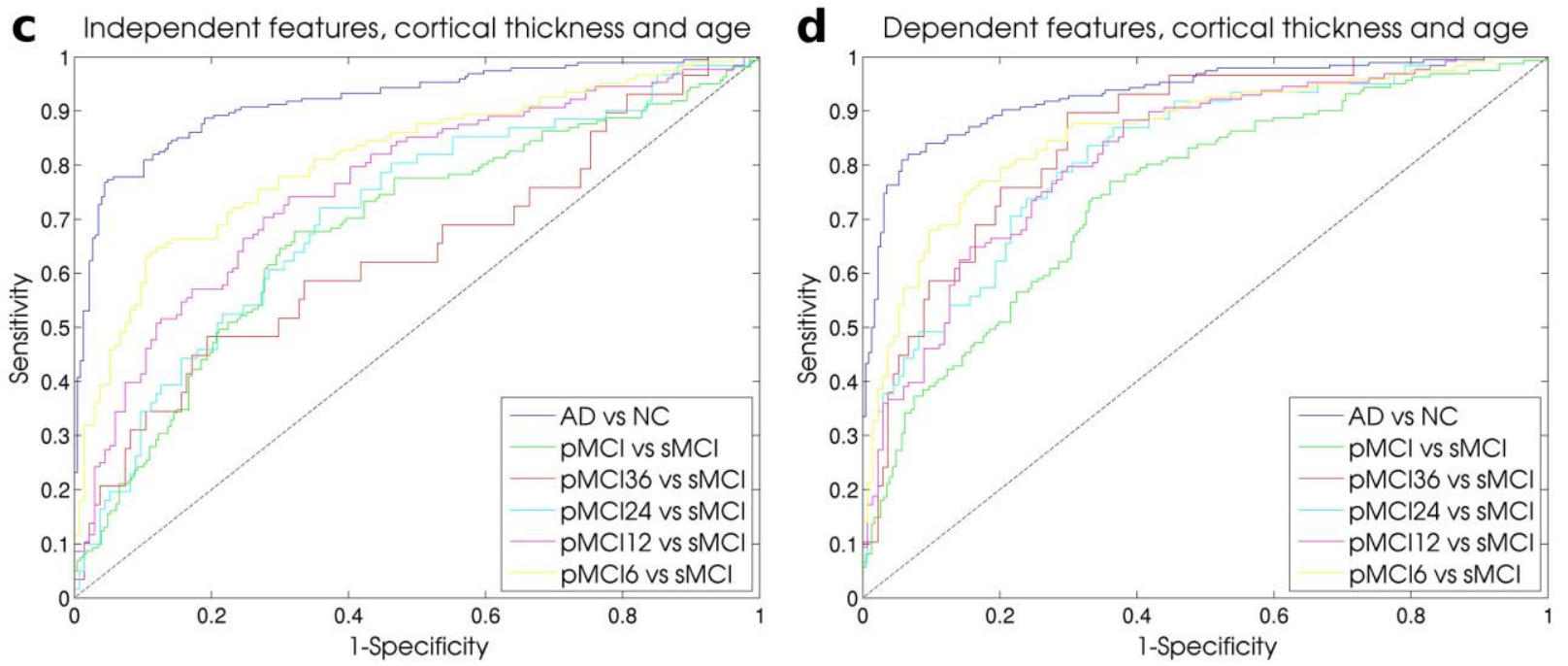

Figure 4. ROC curves for the six classification problems by leave-one-out using independent (a, c) and dependent (b, d) feature sets for cortical thickness alone $(a, b)$ and in combination with age $(c, d)$.

Table 6. P-values for testing true differences in AUC for classifiers with independent feature sets including age. Values found by unpaired, two-sided non-parametric test (Delong et al., 1988). Values below the significance threshold of $5 \%$ are in bold.

\begin{tabular}{|c|c|c|c|c|c|c|}
\hline & & $\begin{array}{l}\mathrm{pMCl} 36 \text { vs } \\
\mathrm{sMCl}\end{array}$ & $\begin{array}{l}\mathrm{pMCl} 24 \text { vs } \\
\mathrm{sMCl}\end{array}$ & $\begin{array}{l}\mathrm{pMCl} 12 \text { vs } \\
\mathrm{sMCl}\end{array}$ & $\begin{array}{l}\text { pMCl6 vs } \\
\text { sMCl }\end{array}$ & $\begin{array}{l}\text { pMCl† vs } \\
\text { sMClt }\end{array}$ \\
\hline & AUC & 63.7 & 70.7 & 76.3 & 81.1 & 68.2 \\
\hline $\begin{array}{l}\mathrm{pMCl} 36 \text { vs } \\
\mathrm{sMCl}\end{array}$ & 63.7 & - & - & - & - & 0.512 \\
\hline $\begin{array}{l}\mathrm{pMCl} 24 \text { vs } \\
\mathrm{sMCl}\end{array}$ & 70.7 & 0.344 & - & - & - & 0.605 \\
\hline $\begin{array}{l}\mathrm{pMCl} 12 \text { vs } \\
\mathrm{sMCl}\end{array}$ & 76.3 & 0.070 & 0.272 & - & - & 0.048 \\
\hline pMCl6 vs sMCl & 81.1 & 0.011 & 0.034 & 0.226 & - & 0.001 \\
\hline$A D$ vs $C N$ & 92.0 & $<0.001$ & $<0.001$ & $<0.001$ & $<0.001$ & $<0.001$ \\
\hline
\end{tabular}

+ Cohort defined as in (Wolz et al., 2011) 
The prediction of conversion from baseline using the combined maximum posterior probability from the four stratified classifiers resulted in an accuracy of $73.5 \%$ (sensitivity $=63.8 \%$, specificity $=84.3 \%$ ) for the three year timeframe (Fig. 5). The figure shows the prediction statistics for the question "will a subject with $\mathrm{MCl}$ convert to $\mathrm{AD}$ within $\mathrm{X}$ months". As the figure indicates, it is more difficult to detect converters within a small time frame (six months) than a large time frame (3 years). This should not be confused with the classification results from the time-homogenized data. Importantly, the combined classifier demonstrates improved prediction accuracy $(73.5 \%)$ over the conventional classifier $(67.8 \%)$ for the three year timeframe.

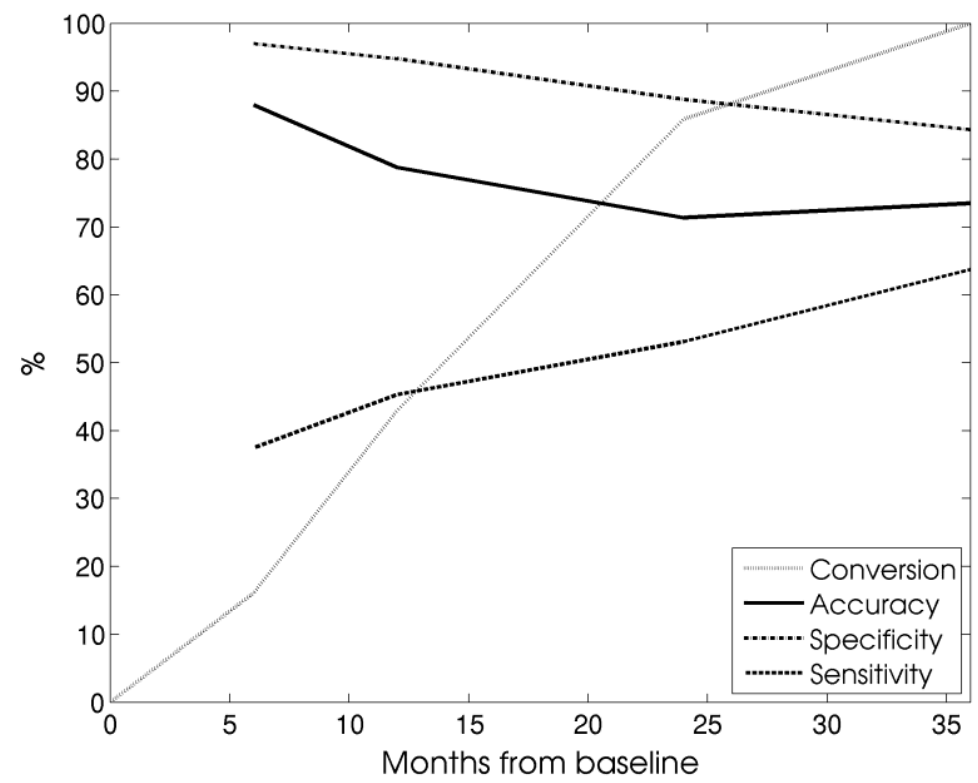

Figure 5. Performance of the combined stratified classifier. The "Conversion" shows the known conversion among pMCI ( $N=149$ ). The accuracy, specificity, and sensitivity show the performance of predicting AD within $X$ months from baseline among the pooled group of $\mathrm{MCl}$ patients $(\mathrm{N}=283)$. The 36 months prediction yields an accuracy of $73.5 \%$.

\section{Discussion}

The homogenization of the $\mathrm{pMCl}$ with respect to "time-to-conversion" led to highly unequal sample sizes. Therefore, the metric of classifier accuracy is difficult to compare directly. A better, single metric of classifier performance is AUC. Thus, we evaluate the performance of the classifiers based on AUC.

Using independent feature sets and cortical thickness we obtained prediction accuracies in the range of $67 \%-76 \%$ and AUCs in the range of $64 \%-81 \%$ (Table 4). Surprisingly, the accuracy for the pMCI36 prediction is nominally higher than the $\mathrm{pMCl} 24$ prediction. However, this is most likely an effect of the reduced sample size for the $\mathrm{pMCl} 36$, as the sensitivity and $\mathrm{AUC}$ are lower for the $\mathrm{pMCl} 36$ prediction.

As expected, the sensitivity and the AUC increase as the time to AD diagnosis is reduced. According to the McNemar's test, only the prediction of pMCl less than 12 months prior to diagnosis (short-term 
prediction) is significantly better than a random classifier when evaluating the independent feature sets. However, when adding the age information all predictions become significantly better than random and the AUCs are improved except for the conventional grouping of $\mathrm{pMCl}$ (Table 5). In terms of AUC, the improvements by adding age are not significant ( $>0.06)$, except for the $A D$ vs. $C N$ classification $(p=0.03)$.

Compared to previously published studies, which use cortical thickness for predicting $\mathrm{pMCl}$ in the ADNI cohort (Cho et al., 2012; Cuingnet et al., 2011; Querbes et al., 2009; Wolz et al., 2011), our prediction accuracies are partly overlapping. We obtain higher accuracies for short-term prediction (<12 months) than previous studies. In contrast, our results for long-term prediction ( $>12$ months) are similar to the results from previously published studies. Unfortunately, these studies did not report AUC, which makes it difficult to compare the true performance of the classifiers due to the unequal sample sizes. Our efforts of homogenising data by "time to conversion" did not have the expected effect on the long-term prediction. It seems that the increased predictive power at the short-term may be attributed to the progression of the disease, which yields a more consistent pattern of cortical neurodegeneration. This is illustrated by the $\mathrm{pMCl} 6$ classifier, which has an AUC of $81 \%$ and performs significantly better than both the long-term prediction $(\mathrm{p}<0.05)$ and the classifier using the conventional groupings of $\mathrm{pMCl}$ and $\mathrm{sMCl}$ $(p=0.001)$. Another perhaps more important factor is the relatively small samples available to evaluate the long-term predictions. The statistical maps, which are the basis for the feature generation, are more affected by noise in the case of small sample sizes. This is seen by the large impact on the classifier performance for the long-term prediction when including the test subject in the statistical map calculation, i.e. double dipping. The AUC increases by 22 percentage points for pMCl36 and 11 percentage points for $\mathrm{pMCl} 24$ predictions when generating dependent feature sets (Table 5). With the continuation of $A D N I$, the number of long-term converters will increase and more consistent feature patterns can be generated for these groups.

All prediction problems had increased AUCs and accuracies when the test subject was included in the calculation of the statistical maps. This illustrates the dangers of double dipping. Even for large sample cases, where one would expect that a single subject has insignificant impact, both the AUC and accuracy is increased by several percentage points. Thus, it is difficult to compare with published prediction results where training and test sets are not independent (Koikkalainen et al., 2011; Querbes et al., 2009; Wolz et al., 2011). However, all our prediction accuracies with dependent feature sets are similar or better than all previously published results, except for Misra et al. (2009), where only $27 \mathrm{pMCl}$ subjects 
were tested (Table 1). The AUC reported in Misra et al. (2009) is similar to our 12 months prediction, but lower than our 6 months prediction. These results highlight the competitive prediction accuracy obtained by the proposed method.

Compared to the conventional grouping of $\mathrm{pMCl}$ and $\mathrm{sMCl}$ as published in Wolz et al. (2011), only the independent $\mathrm{pMCl} 24$ prediction performed worse (67.2\% vs. 67.8\%). This is an indication of the improved accuracy by homogenizing data. However, due to the unequal sample sizes and the absence of AUC statistics, the improvements cannot with certainty be attributed to the homogenization. In the head to head comparison using the exact same groupings, we obtained an accuracy of $68 \%$ using independent feature sets. This is the same accuracy as the combined multivariate prediction based on manifold learning, hippocampal volume, cortical thickness and TBM (68\%), but higher than using cortical thickness alone (58\%) as published by Wolz et al. (2011). It should be noted that the features Wolz and colleagues used in the cortical thickness and TBM analyses were dependent, i.e. double dipping occurred. When we applied our dependent feature sets, we obtained an accuracy of $71 \%$.

The classifier performance obtained for the $\mathrm{pMCl}$ sub-groups demonstrates a theoretical improvement by the time-homogenization. In practice, predictions must be made on subjects with variable time to conversion. Our attempt at using the stratified classifiers in a clinically relevant way demonstrated that we could predict conversion from baseline within three years with an accuracy of $74 \%$. While the specificity of this prediction was high (84\%), the sensitivity was relatively low (64\%). This may be due to the comparison of posterior probability across classifiers. Considering the uncertainties of the classifiers and the fact that the outcome "sMCl" is possible in all four classifiers, this way of combining the stratified classifiers may be conservative with respect to predicting $\mathrm{pMCl}$. Other ways of combining the classifiers may provide more balanced sensitivity and specificity.

With these new methods, superior prediction accuracies can be obtained. The specificity was higher than the sensitivity for the long-term prediction. From an economic perspective a high specificity is very important in clinical trials when recruiting subjects. Reducing the number of false positives in trials may save time and reduce the associated costs. On the other hand, the sensitivity for the long-term prediction lags behind. Here, clearly more research needs to be done to improve the technique, and to be able to benefit from eventual neuroprotective therapies. 


\section{Image processing}

In this study we used a combination of highly consistent and robust image processing methods to measure the cortical thickness. Previous studies using cortical thickness have suffered from high exclusion rates due weak links in the image processing pipeline (Cuingnet et al., 2011; Eskildsen et al., 2011c; Wolz et al., 2011). In our experience, the main factor for excluding scans due to processing is the skull stripping step. By using BEaST, a recent robust brain extraction algorithm (Eskildsen et al., 2011a), we were able to effectively reduce the exclusion rate. In our study we excluded only $2.7 \%$ of all scans, which, to the best of our knowledge, is the lowest exclusion rate of any published cortical thickness analysis on ADNI data. The low exclusion rate enabled us to construct relatively large samples of $\mathrm{pMCl}$ subjects homogenous with respect to time to conversion. Furthermore, our strategy of denoising the images before cortical surface extraction has been shown to provide more accurate results than processing unfiltered images (Eskildsen et al., 2011b).

\section{Regions selected for prediction model}

The ROls most often chosen by the feature selection for prediction indicate which anatomical regions are involved at different times prior to the progression from $\mathrm{MCl}$ to $\mathrm{AD}$. As shown in Table 3, medial temporal lobe structures are selected in all prediction problems. Hippocampus is included in all predictions except the $\mathrm{pMCl} 36$, while the right parahippocampal gyrus is used in all cases. The parahippocampal gyrus has previously been found to be highly discriminative for separating $A D$ patients from healthy controls using cortical thickness and multivariate analysis, however in the absence of hippocampal segmentation (Lerch et al., 2008). We speculate that a possible explanation for the specific timeframe observed here might have to do with the proposed progression of the disease. Histological studies suggest that the integrity of the entorhinal cortex (part of the parahippocampal gyrus) is among the first affected, which is then only later followed by an atrophy of the hippocampus (Braak et al., 1993). The fact that at 36 month prior to diagnosis the hippocampus is not yet a discriminative factor in the MRI based analysis would thus nicely complement these histological findings. In addition to medial temporal lobe structures, ROIs were mostly selected from the cingulate gyrus. The cingulate gyrus is well known to be affected in early AD. What is surprising is the selection of ROIs in the occipital lobe, mostly the left, for all pMCl predictions, but not for the $A D$ vs. CN classification. This suggests that the occipital lobes might also be affected early in the disease process, but that it might not be specific at later stages in the disease process. However, other studies have reported cortical thinning in the occipital lobe in AD recently as well (Hartikainen et al., 2012), and posterior cortical atrophy in general is associated with a 
range of different pathologies, including $A D$ (Crutch et al., 2012). In fact, posterior atrophy may aid differentiation of $A D$ from frontotemporal lobar degeneration (Lehmann et al., 2012). The lack of selection of these regions for the $A D$ vs. $C N$ classification problem may be caused by the highly significant differences of medial temporal regions rendering the more posterior regions superfluous for the classifier.

\section{Feature generation}

The feature generation approach used in our study is similar in spirit to the approach suggested by Fan et al. (2007), who used a watershed algorithm on the VBM-based statistical parametric map to select ROIs. Using a watershed algorithm on the statistical maps generated by cortical thickness would yield less compact ROls, as the regions with high t-values often are elongated following a sulcus or gyrus across several anatomical regions. In the proposed method we sought more focal features and therefore applied the constrained region growing region as described above. We acknowledge that our method for feature generation is just one among many possibilities using the statistical map. Nevertheless, we expect the tendency of increased sensitivity by homogenising the data by time to conversion will remain the same irrespective of the feature generation method applied.

\section{Limitations}

One of the main limitations in any AD study involving in vivo data, is the uncertainty of the diagnosis. That is the reason why the diagnosis will always be "probable $A D$ " until an autopsy can confirm the diagnosis. An optimistic estimate of the correct diagnosis rate using the accepted $A D$ criteria is approximately 90\% (Ranginwala et al., 2008). Even if AD type dementia is indeed present, the picture is often distorted by other dementia types, such as vascular dementia. Thus, the patterns of neurodegeneration, found in studies like the present, will have uncertainties due to the inherent uncertainty from difficulties in assigning correct diagnoses.

Another problem related to diagnosis, is the term "conversion". In this study, as in many other studies, we have accepted the term "conversion" as an individual suffering from mild cognitive impairment who then fulfils the AD diagnostic criteria at a subsequent clinical visit. The progression from mild symptoms to a state where the diagnostic criteria are fulfilled is a gradual decline and not an abrupt change. Thus the actual disease stage at time of diagnosis will be different from individual to individual. Our efforts in trying to stage the progression by "time to conversion" may be futile due to the fuzziness of the 
conversion term. However, it is currently the best we can do to compensate for the noise introduced by pooling all converters.

Another nuisance variable is the uncertainty of the label "stable $\mathrm{MCl}$ ". Even though stable $\mathrm{MCl}$ do not progress to $A D$ within the follow-up period included in the ADNI study, we do not know if they progress to another type of dementia, which makes the classification harder as there may be overlapping patterns of structural changes. In other studies (Westman et al., 2011; Wolz et al., 2011), authors have simply generated their group of $\mathrm{sMCl}$ as the complementary of $\mathrm{pMCl}$ even including subjects dropping out after baseline examinations. Here, we tried to homogenize our stable group by requiring a diagnosis of $\mathrm{MCl}$ for at least three years. However, the uncertainty of influence from other pathologies still exists.

As described in the introduction, many studies have used data from the ADNI cohort to evaluate various imaging markers and investigate the progression of AD. The question is if results obtained on ADNI data can be transferred to other populations. First, the female / male ratio in the ADNI cohort is poor with almost twice as many males as females. Second, the recruitment of $\mathrm{MCl}$ patients in ADNI tended to select "late $\mathrm{MCl}$ " subjects. These issues may make it difficult to transfer the results to other cohorts. Westman et al. (2011) found similar patterns in ADNI and the European study, AddNeuroMed. However, their prediction accuracy could not be transferred from one cohort to the other. Nevertheless, ADNI remains to be an excellent initiative enabling the comparison of methods across institutions. Furthermore, the continuing ADNI study enrols new patients, and the ADNI GO and ADNI 2 projects will

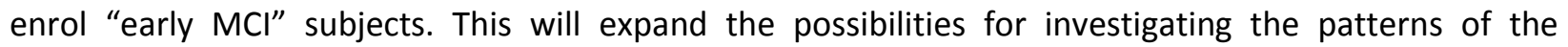
incipient neurodegeneration in AD.

\section{Conclusion}

Using patterns of characteristic cortical thinning in disease stages of progressive $\mathrm{MCl}$ compared to $\mathrm{MCl}$ patients who remained stable for three years demonstrated promising results for the prediction of patients with prodromal AD progressing to probable AD. By avoiding double dipping we obtained a more accurate and unbiased estimate of the predictive power of cortical thickness measurements than published to date. The prediction accuracies obtained by subgrouping progressive $\mathrm{MCl}$ patients with respect to "time to conversion" were better than previously published results on the same cohort. The improved accuracies are likely caused by this homogenization and improvements in the image processing pipeline in terms of robustness and accuracy. The results demonstrate a theoretical 
improvement and our preliminary experiments show that clinically relevant predictions may be improved by learning time-homogenized patterns.

The experiments showed that prediction accuracies can be artificially inflated by including the subject under analysis in the generation of the discriminant features. One should therefore be careful to avoid double dipping in the estimation of classifications and predictions based on cortical thickness statistical maps.

The specificity of predicting whether an $\mathrm{MCl}$ subject converts to $A D$ within three years from baseline was relatively high (84\%), while the sensitivity was relatively low (64\%). To be clinically applicable the sensitivity for the long-term prediction needs to be improved to be able to benefit from eventual neuroprotective therapies. The relatively high specificity for the long-term prediction holds promises of reduced costs associated with recruiting subjects for clinical trials.

\section{Acknowledgements}

Data collection and sharing for this project was funded by the Alzheimer's Disease Neuroimaging Initiative (ADNI) (National Institutes of Health Grant U01 AG024904). ADNI is funded by the National Institute on Aging, the National Institute of Biomedical Imaging and Bioengineering, and through generous contributions from the following: Abbott, AstraZeneca AB, Bayer Schering Pharma AG, BristolMyers Squibb, Eisai Global Clinical Development, Elan Corporation, Genentech, GE Healthcare, GlaxoSmithKline, Innogenetics, Johnson and Johnson, Eli Lilly and Co., Medpace, Inc., Merck and Co., Inc., Novartis AG, Pfizer Inc, F. Hoffman-La Roche, Schering-Plough, Synarc, Inc., as well as non-profit partners the Alzheimer's Association and Alzheimer's Drug Discovery Foundation, with participation from the U.S. Food and Drug Administration. Private sector contributions to ADNI are facilitated by the Foundation for the National Institutes of Health (www.fnih.org). The grantee organization is the Northern California Institute for Research and Education, and the study is coordinated by the Alzheimer's Disease Cooperative Study at the University of California, San Diego. ADNI data are disseminated by the Laboratory for Neuro Imaging at the University of California, Los Angeles. This research was also supported by NIH grants P30AG010129, K01 AG030514, and the Dana Foundation. The authors wish to thank Dr. Robin Wolz for supplying the list of subjects used in Wolz et al. (2011), thus enabling a direct comparison between methods. 


\section{References}

Apostolova, L.G., Thompson, P.M., 2008. Mapping progressive brain structural changes in early Alzheimer's disease and mild cognitive impairment. Neuropsychologia 46, 1597-1612.

Braak, H., Braak, E., 1991. Neuropathological stageing of Alzheimer-related changes. Acta Neuropathol 82, 239-259.

Braak, H., Braak, E., Bohl, J., 1993. Staging of Alzheimer-related cortical destruction. Eur Neurol 33, 403408.

Cho, Y., Seong, J.K., Jeong, Y., Shin, S.Y., 2012. Individual subject classification for Alzheimer's disease based on incremental learning using a spatial frequency representation of cortical thickness data. Neurolmage 59, 2217-2230.

Chupin, M., Gerardin, E., Cuingnet, R., Boutet, C., Lemieux, L., Lehericy, S., Benali, H., Garnero, L., Colliot, O., 2009. Fully automatic hippocampus segmentation and classification in Alzheimer's disease and mild cognitive impairment applied on data from ADNI. Hippocampus 19, 579-587.

Collins, D.L., Neelin, P., Peters, T.M., Evans, A.C., 1994. Automatic 3D intersubject registration of MR volumetric data in standardized Talairach space. Journal of computer assisted tomography 18, 192-205. Coupe, P., Eskildsen, S.F., Manjon, J.V., Fonov, V.S., Collins, D.L., 2012. Simultaneous segmentation and grading of anatomical structures for patient's classification: application to Alzheimer's disease.

Neurolmage 59, 3736-3747.

Coupe, P., Manjon, J.V., Gedamu, E., Arnold, D., Robles, M., Collins, D.L., 2010. Robust Rician noise estimation for MR images. Med Image Anal 14, 483-493.

Coupe, P., Yger, P., Prima, S., Hellier, P., Kervrann, C., Barillot, C., 2008. An optimized blockwise nonlocal means denoising filter for 3-D magnetic resonance images. IEEE transactions on medical imaging 27 , 425-441.

Crutch, S.J., Lehmann, M., Schott, J.M., Rabinovici, G.D., Rossor, M.N., Fox, N.C., 2012. Posterior cortical atrophy. Lancet Neurol 11, 170-178.

Cuingnet, R., Gerardin, E., Tessieras, J., Auzias, G., Lehericy, S., Habert, M.O., Chupin, M., Benali, H., Colliot, O., 2011. Automatic classification of patients with Alzheimer's disease from structural MRI: a comparison of ten methods using the ADNI database. Neurolmage 56, 766-781.

Davatzikos, C., Bhatt, P., Shaw, L.M., Batmanghelich, K.N., Trojanowski, J.Q., 2011. Prediction of MCl to AD conversion, via MRI, CSF biomarkers, and pattern classification. Neurobiology of aging 32, 2322 e2319-2327.

DeLong, E.R., DeLong, D.M., Clarke-Pearson, D.L., 1988. Comparing the areas under two or more correlated receiver operating characteristic curves: a nonparametric approach. Biometrics 44, 837-845. Ellis, K.A., Bush, A.I., Darby, D., De Fazio, D., Foster, J., Hudson, P., Lautenschlager, N.T., Lenzo, N., Martins, R.N., Maruff, P., Masters, C., Milner, A., Pike, K., Rowe, C., Savage, G., Szoeke, C., Taddei, K., Villemagne, V., Woodward, M., Ames, D., 2009. The Australian Imaging, Biomarkers and Lifestyle (AIBL) study of aging: methodology and baseline characteristics of 1112 individuals recruited for a longitudinal study of Alzheimer's disease. Int Psychogeriatr 21, 672-687.

Eskildsen, S.F., Coupe, P., Fonov, V., Manjon, J.V., Leung, K.K., Guizard, N., Wassef, S.N., Ostergaard, L.R., Collins, D.L., 2011a. BEaST: Brain extraction based on nonlocal segmentation technique. Neurolmage. Eskildsen, S.F., Coupe, P., Fonov, V., Østergaard, L.R., Collins, D.L., 2011b. Effect of non-local means denoising on cortical segmentation accuracy with FACE. 17th Annual Meeting of the Organization on Human Brain Mapping, Quebec City, p. 673.

Eskildsen, S.F., Fonov, V., Coupé, P., Østergaard, L.R., Collins, D.L., 2011c. Prediction of Alzheimer's Disease in Subjects with Mild Cognitive Impairment Using Structural Patterns of Cortical Thinning*. In: Dremstrup, K., Rees, S., Jensen, M. $\varnothing$. (Eds.), 15th Nordic-Baltic Conference on Biomedical Engineering and Medical Physics (NBC 2011). Springer Berlin Heidelberg, pp. 156-159. 
Eskildsen, S.F., Ostergaard, L.R., 2006. Active surface approach for extraction of the human cerebral cortex from MRI. Medical image computing and computer-assisted intervention : MICCAI ... International Conference on Medical Image Computing and Computer-Assisted Intervention 9, 823-830. Fan, Y., Shen, D., Gur, R.C., Gur, R.E., Davatzikos, C., 2007. COMPARE: classification of morphological patterns using adaptive regional elements. IEEE transactions on medical imaging 26, 93-105. Fonov, V., Evans, A.C., Botteron, K., Almli, C.R., McKinstry, R.C., Collins, D.L., 2011. Unbiased average age-appropriate atlases for pediatric studies. Neurolmage 54, 313-327.

Frisoni, G.B., Fox, N.C., Jack, C.R., Jr., Scheltens, P., Thompson, P.M., 2010. The clinical use of structural MRI in Alzheimer disease. Nature reviews. Neurology 6, 67-77.

Hartikainen, P., Rasanen, J., Julkunen, V., Niskanen, E., Hallikainen, M., Kivipelto, M., Vanninen, R., Remes, A.M., Soininen, H., 2012. Cortical Thickness in Frontotemporal Dementia, Mild Cognitive Impairment, and Alzheimer's Disease. Journal of Alzheimer's disease : JAD 30, 857-874.

Jack, C.R., Jr., Barkhof, F., Bernstein, M.A., Cantillon, M., Cole, P.E., Decarli, C., Dubois, B., Duchesne, S., Fox, N.C., Frisoni, G.B., Hampel, H., Hill, D.L., Johnson, K., Mangin, J.F., Scheltens, P., Schwarz, A.J., Sperling, R., Suhy, J., Thompson, P.M., Weiner, M., Foster, N.L., 2011. Steps to standardization and validation of hippocampal volumetry as a biomarker in clinical trials and diagnostic criterion for Alzheimer's disease. Alzheimer's \& dementia : the journal of the Alzheimer's Association 7, 474-485 e474.

Koikkalainen, J., Lotjonen, J., Thurfjell, L., Rueckert, D., Waldemar, G., Soininen, H., 2011. Multi-template tensor-based morphometry: application to analysis of Alzheimer's disease. Neurolmage 56, 1134-1144. Kriegeskorte, N., Simmons, W.K., Bellgowan, P.S., Baker, C.I., 2009. Circular analysis in systems neuroscience: the dangers of double dipping. Nat Neurosci 12, 535-540.

Lehmann, M., Koedam, E.L., Barnes, J., Bartlett, J.W., Ryan, N.S., Pijnenburg, Y.A., Barkhof, F., Wattjes, M.P., Scheltens, P., Fox, N.C., 2012. Posterior cerebral atrophy in the absence of medial temporal lobe atrophy in pathologically-confirmed Alzheimer's disease. Neurobiology of aging 33, 627 e621-627 e612. Lerch, J.P., Pruessner, J., Zijdenbos, A.P., Collins, D.L., Teipel, S.J., Hampel, H., Evans, A.C., 2008. Automated cortical thickness measurements from MRI can accurately separate Alzheimer's patients from normal elderly controls. Neurobiology of aging 29, 23-30.

Lovestone, S., Francis, P., Kloszewska, I., Mecocci, P., Simmons, A., Soininen, H., Spenger, C., Tsolaki, M., Vellas, B., Wahlund, L.O., Ward, M., 2009. AddNeuroMed--the European collaboration for the discovery of novel biomarkers for Alzheimer's disease. Ann N Y Acad Sci 1180, 36-46.

Lupien, S.J., Evans, A., Lord, C., Miles, J., Pruessner, M., Pike, B., Pruessner, J.C., 2007. Hippocampal volume is as variable in young as in older adults: implications for the notion of hippocampal atrophy in humans. Neurolmage 34, 479-485.

McKhann, G., Drachman, D., Folstein, M., Katzman, R., Price, D., Stadlan, E.M., 1984. Clinical diagnosis of Alzheimer's disease: report of the NINCDS-ADRDA Work Group under the auspices of Department of Health and Human Services Task Force on Alzheimer's Disease. Neurology 34, 939-944.

Misra, C., Fan, Y., Davatzikos, C., 2009. Baseline and longitudinal patterns of brain atrophy in $\mathrm{MCl}$ patients, and their use in prediction of short-term conversion to AD: results from ADNI. Neurolmage 44, 1415-1422.

Mueller, S.G., Weiner, M.W., Thal, L.J., Petersen, R.C., Jack, C., Jagust, W., Trojanowski, J.Q., Toga, A.W., Beckett, L., 2005. The Alzheimer's disease neuroimaging initiative. Neuroimaging clinics of North America 15, 869-877, xi-xii.

Narr, K.L., Bilder, R.M., Toga, A.W., Woods, R.P., Rex, D.E., Szeszko, P.R., Robinson, D., Sevy, S., GunduzBruce, H., Wang, Y.P., DeLuca, H., Thompson, P.M., 2005. Mapping cortical thickness and gray matter concentration in first episode schizophrenia. Cerebral cortex 15, 708-719. 
Peng, H., Long, F., Ding, C., 2005. Feature selection based on mutual information: criteria of maxdependency, max-relevance, and min-redundancy. IEEE transactions on pattern analysis and machine intelligence 27, 1226-1238.

Plant, C., Teipel, S.J., Oswald, A., Bohm, C., Meindl, T., Mourao-Miranda, J., Bokde, A.W., Hampel, H., Ewers, M., 2010. Automated detection of brain atrophy patterns based on MRI for the prediction of Alzheimer's disease. Neurolmage 50, 162-174.

Querbes, O., Aubry, F., Pariente, J., Lotterie, J.A., Demonet, J.F., Duret, V., Puel, M., Berry, I., Fort, J.C., Celsis, P., 2009. Early diagnosis of Alzheimer's disease using cortical thickness: impact of cognitive reserve. Brain : a journal of neurology 132, 2036-2047.

Ranginwala, N.A., Hynan, L.S., Weiner, M.F., White, C.L., 3rd, 2008. Clinical criteria for the diagnosis of Alzheimer disease: still good after all these years. Am J Geriatr Psychiatry 16, 384-388.

Ritchie, K., Lovestone, S., 2002. The dementias. Lancet 360, 1759-1766.

Robin, X., Turck, N., Hainard, A., Tiberti, N., Lisacek, F., Sanchez, J.C., Muller, M., 2011. pROC: an opensource package for $R$ and $S+$ to analyze and compare ROC curves. BMC bioinformatics 12, 77.

Shin, Y.W., Yoo, S.Y., Lee, J.K., Ha, T.H., Lee, K.J., Lee, J.M., Kim, I.Y., Kim, S.I., Kwon, J.S., 2007. Cortical thinning in obsessive compulsive disorder. Human brain mapping 28, 1128-1135.

Sled, J.G., Zijdenbos, A.P., Evans, A.C., 1998. A nonparametric method for automatic correction of intensity nonuniformity in MRI data. IEEE transactions on medical imaging 17, 87-97.

Tzourio-Mazoyer, N., Landeau, B., Papathanassiou, D., Crivello, F., Etard, O., Delcroix, N., Mazoyer, B., Joliot, M., 2002. Automated anatomical labeling of activations in SPM using a macroscopic anatomical parcellation of the MNI MRI single-subject brain. Neurolmage 15, 273-289.

Vemuri, P., Whitwell, J.L., Kantarci, K., Josephs, K.A., Parisi, J.E., Shiung, M.S., Knopman, D.S., Boeve, B.F., Petersen, R.C., Dickson, D.W., Jack, C.R., Jr., 2008. Antemortem MRI based STructural Abnormality iNDex (STAND)-scores correlate with postmortem Braak neurofibrillary tangle stage. Neurolmage 42, 559-567. Westman, E., Simmons, A., Muehlboeck, J.S., Mecocci, P., Vellas, B., Tsolaki, M., Kloszewska, I., Soininen, H., Weiner, M.W., Lovestone, S., Spenger, C., Wahlund, L.O., 2011. AddNeuroMed and ADNI: similar patterns of Alzheimer's atrophy and automated MRI classification accuracy in Europe and North America. Neurolmage 58, 818-828.

Wolz, R., Julkunen, V., Koikkalainen, J., Niskanen, E., Zhang, D.P., Rueckert, D., Soininen, H., Lotjonen, J., 2011. Multi-method analysis of MRI images in early diagnostics of Alzheimer's disease. PloS one 6, e25446.

Yoon, U., Lee, J.M., Im, K., Shin, Y.W., Cho, B.H., Kim, I.Y., Kwon, J.S., Kim, S.I., 2007. Pattern classification using principal components of cortical thickness and its discriminative pattern in schizophrenia. Neurolmage 34, 1405-1415.

Yuan, Y., Gu, Z.X., Wei, W.S., 2009. Fluorodeoxyglucose-positron-emission tomography, single-photon emission tomography, and structural MR imaging for prediction of rapid conversion to Alzheimer disease in patients with mild cognitive impairment: a meta-analysis. AJNR Am J Neuroradiol 30, 404-410. 